\title{
Three-dimensional digitizing and anatomic study of lumbar vertebral canal and pedicle in children
}

\author{
Xing Wang ${ }^{1,2}$, Shao-Jie Zhang ${ }^{1,2}$, Yuan-Zhi Zhang ${ }^{2,3}$, Xiao-He Li $^{1}$, Zhi-Feng Zhang ${ }^{4}$, Chao-Qun Wang ${ }^{5}$, Shang Gao ${ }^{1}$, \\ Jun Shi ${ }^{6}$, Zhi-Jun Li', \\ ${ }^{1}$ Teaching and Researching Institute of Human Anatomy, School of Basic Medicine, Hohhot, China \\ ${ }^{2}$ Digital Medical Center, School of Basic Medicine, Inner Mongolia Medical University, Hohhot, China \\ ${ }^{3}$ Department of Orthopedics, the Affiliated Hospital, Inner Mongolia Medical University, Hohhot, China \\ ${ }^{4}$ Department of Joint Surgery, the Second Affiliated Hospital, Inner Mongolia Medical University, Hohhot, China \\ ${ }^{5}$ Department of Radiology, the Affiliated Hospital, Inner Mongolia Medical University, Hohhot, China \\ ${ }^{6}$ Teaching and Researching Institute of Physiology, School of Basic Medicine, Inner Mongolia Medical University, Hohhot, China
}

Videosurgery Miniinv 2018; 13 (4): 518-524 DOI: https://doi.org/10.5114/wiitm.2018.77554

\begin{abstract}
Introduction: Spinal pedicle screw internal fixation has been widely used in adult spine injury fixation. Due to being in a period of continuous growth and development, the spine of children at different ages shows different characteristics from adults in terms of anatomy, physiological function, and biomechanics. Furthermore, because the pedicle of children is small, has large anatomic variation, and has complex adjacent relationships, the surgical risk is extremely high. How to improve the screwing accuracy is the key to the success of children's pedicle internal fixation. Therefore, applying the concept of digitized and individualized screwing will be of great significance to children's pedicle screwing. Aim: To investigate the morphologies, development patterns, and aging characteristics of the lumbar vertebral pedicle (LVP) in children aged 6-11 years, and to provide a theoretical basis for screw implantation and related biomechanical studies.

Material and methods: A total of 60 children aged 6-11 years were selected for the intergroup measurement and statistical analysis of their lumbar diameter, pedicle diameter, screw canal length (SCL), etc.

Results: Generally, the vertebral foramen diameter (ID), sagittal diameter (SD), pedicle width (PW), and SCL as well as the pedicle height $(\mathrm{PH})$ exhibited an increasing trend with age and increasing vertebral sequence among children aged 6-11 years.

Conclusions: By observing the LVP in children using 3D digital reconstruction technology, the morphology of the spinal canal and pedicles at different lumbar segments showed obvious development patterns, and the best treatment protocol should be selected according to the LVP characteristics in clinical applications.
\end{abstract}

Key words: lumbar vertebra, pedicle, morphologic characteristics, 3D reconstruction measurement, children.

\section{Introduction}

With advances in science, pedicle screw fixation technology has developed into a mature spinal surgical technique for treating adolescents and adults with spinal trauma, infectious lesions, degenerative lesions, or neoplastic lesions, and it particularly plays a crucial role in orthopedic surgery for spinal deformity [1-3]. The pedicle system could effectively restore the spinal physiological curvature in the treatment

\section{Address for correspondence}

Zhi-Jun Li, Teaching and Researching Institute of Human Anatomy, School of Basic Medicine, Inner Mongolia Medical University, 5 Xinhua St, Hohhot 010059, China, phone: +86 471 6657551, fax: +86 471 6657551, e-mail: zhijunlicn@126.com Jun Shi, Teaching and Researching Institute of Physiology, School of basic Medicine, Inner Mongolia Medical University, Hohhot O10059, 
of thoracolumbar spine fractures and vertebral fractures and could also achieve good reduction via distraction, thus achieving the purpose of 3D fixation. The spinal pedicle screw fixation technique has been widely used for internal fixation in adult spinal injury for its characteristics of three-point fixation, reasonable biomechanics, and firm stability [4]. Children's spine is in a constant growing stage, and it exhibits certain characteristics of anatomic form, physiologic functions, and biomechanics, different from those in adults at different ages. With increased disease awareness, spinal injuries in children have also received increasing attention from scholars $[5,6]$. However, the relatively mature surgical techniques in adults are restricted when used for treating thoracic and lumbar disorders in children. The main reasons are: (1) spinal disorders in children are different from those in adults, because the spine is at the peak of continuous development; therefore, their morphological structures are very different, the incidence of spinal trauma and tumor is low, while the incidence of spinal deformity and tuberculosis is the highest; (2) pediatric thoracic and lumbar pedicle is smaller than an adult's, so screw implantation would be difficult and have bigger risks, and also there is a lack of specialized lumbar screws for children [7, 8]; (3) further study is still needed on the impact of screw implantation on spinal growth. It was reported abroad that spinal injury in children [9-11] accounted for about $1 \%$ to $10 \%$ of all spinal cord injuries, and because children's pedicle is smaller, anatomic structures vary greatly, adjacent relationships are complex, and the surgical risks are extremely high, improving the accuracy of screw implantation has become the key towards the further development of pedicle screw fixation technology in children. Therefore, the concept of digital individual pedicle screw implantation would have very important significance towards pedia-pedicle screw implantation. Currently, this application has been reported abroad $[12,13]$, but most cases were concentrated on studying pedicle morphologies [14], and the number of studies in China is relatively small. Therefore, the spinal morphologies and related theoretical parameters of children's vertebral column need to be urgently enriched with large samples.

\section{Aim}

This study used imaging and 3D reconstruction technology, and obtained the lumbar vertebral ped- icle (LVP) and screw implantation associated parameters in different-age-group healthy children, aiming to explore its morphological development patterns and to provide a theoretical basis for pedia-pedicle screw design, feasibility and safety range of screw implantation and related biomechanical studies.

\section{Material and methods}

\section{Subjects}

Children who underwent lumbar computed tomography (CT) in the Department of Radiology, the Affiliated Hospital of Inner Mongolia Medical University, were selected, while those with lumbar trauma, deformity, or obvious neurological symptoms and signs were excluded. The data were collected from the children in outpatient clinics, and those with obvious lumbar spine bone fractures, bone fractures, or deformities were excluded. This study did not involve the recruitment of separate volunteers, and such recruitment will be implemented with the approval of the school ethics committee if necessary. The normal radiation dose range of CT is $8.11 \mathrm{mGy}$. A total of 60 children aged 6-11 years (mean: $9.5 \pm 1.6$ years) were selected (boys: 30 , girls: 30 ) and divided into two groups for statistical analysis (Table I). This study was conducted in accordance with the Declaration of Helsinki. This study was conducted with approval from the Ethics Committee of Inner Mongolia Medical University. Written informed consent was obtained from all participants' guardians.

\section{Experimental steps}

The 64-slice CT machine (CE Company) spiral computed tomography (CT) scanner (USA) was used to scan vertebrae from T12 to S1 using the head-to-toe direction; the scanning line was vertical to the body central axis; and the scanning pa-

Table I. Subjects and grouping

\begin{tabular}{|lcccc|}
\hline Group & $\begin{array}{c}\text { Age segment } \\
\text { [years] }\end{array}$ & Cases & \multicolumn{2}{c|}{ Gender } \\
\cline { 3 - 5 } & $6-8$ & 30 & 15 & Girl \\
\hline B & $9-11$ & 30 & 15 & 15 \\
\hline Sum & $6-11$ & 60 & 30 & 30 \\
\hline
\end{tabular}




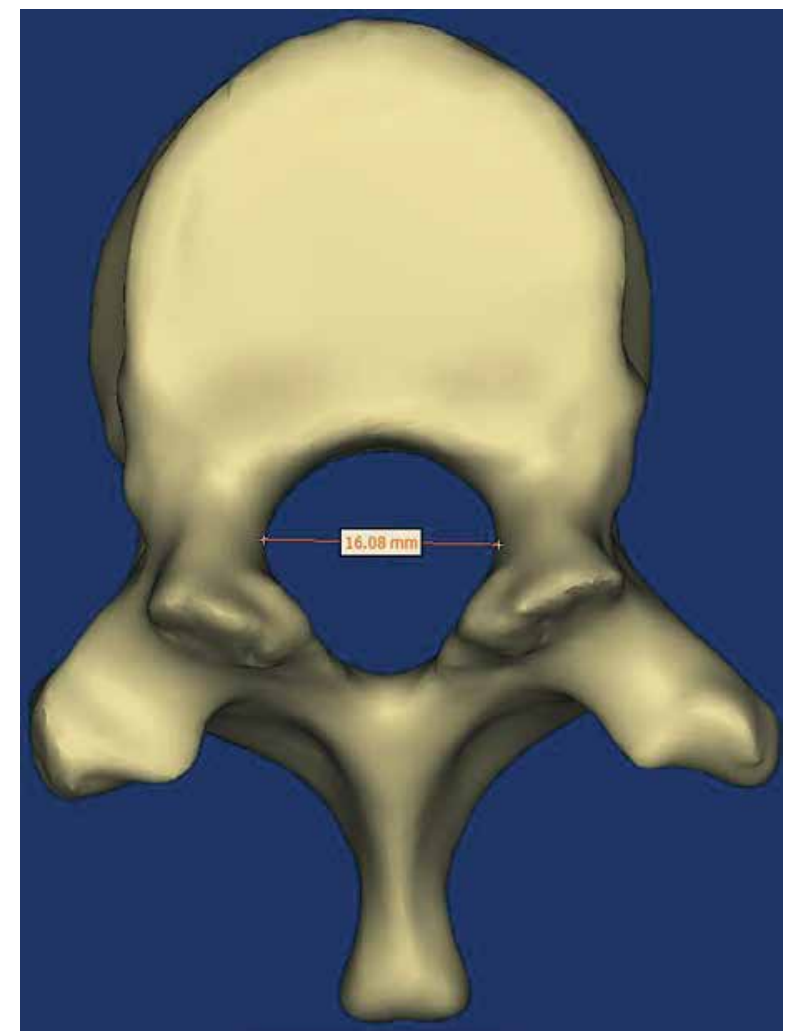

Photo 1. Vertebral foramen diameter (ID)

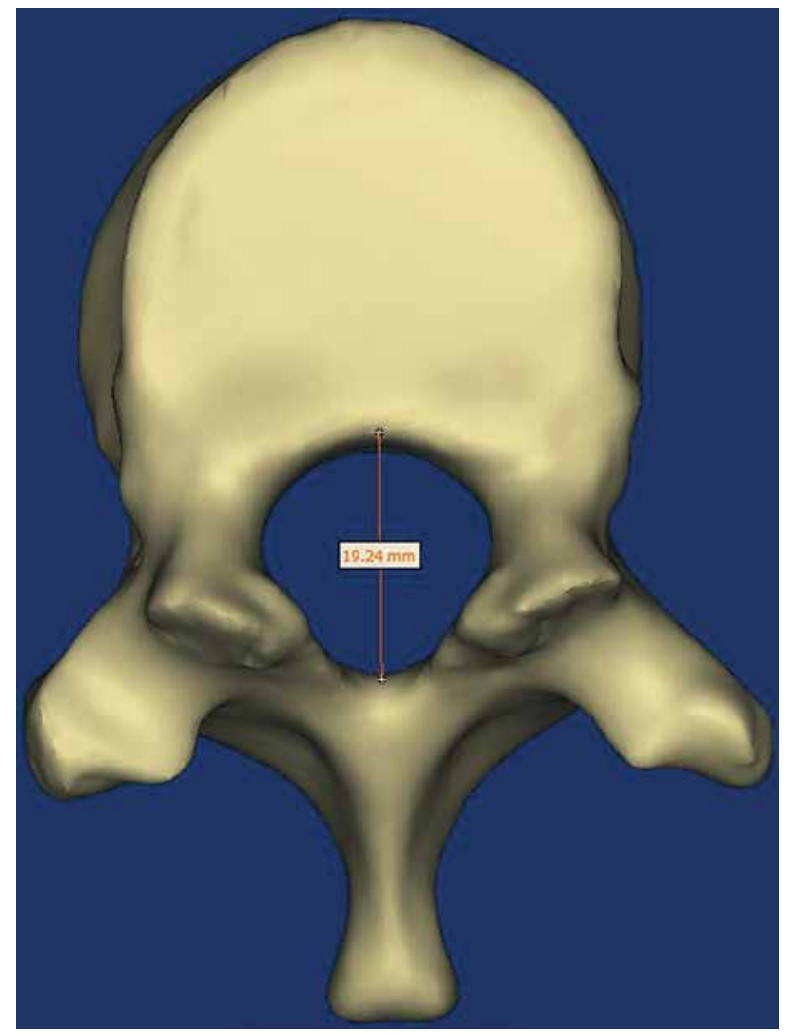

Photo 2. Sagittal diameter (SD) rameters were as follows: slice thickness $1.25 \mathrm{~mm}$, pitch $1.25 \mathrm{~mm}$, thickness of reconstructed layer $0.625 \mathrm{~mm}$, reconstruction pitch $0.625 \mathrm{~mm}$, Fov $30 \times 30 \mathrm{~cm}$, matrix $512 \times 512 \mathrm{dpi}$, tube voltage $150 \mathrm{KV}$ and current $260 \mathrm{~mA}$. The original scanning data were imported into one computer with the DICOM format, and the 3D reconstruction software Mimics 15.0 (Materialise's interactive medical image control system, Materialise, Belgium) was used for post-treatment measurements (provided by the digital Medical Center, Inner Mongolia Medical University).

\section{Measurement parameters}

Vertebral foramen diameter (ID): distance between the left and right edges via the spinal central canal (Photo 1); sagittal diameter (SD): distance between the posterior edge of the longitudinal ligament to the anterior edge of the ligamenta flava (Photo 2); pedicle width (PW): the shortest distance between the edges of inner and outer pedicle cortex (Photo 3); pedicle height ( $\mathrm{PH})$ : the shortest distance between the upper and lower pedicle cortex (Photo 4).

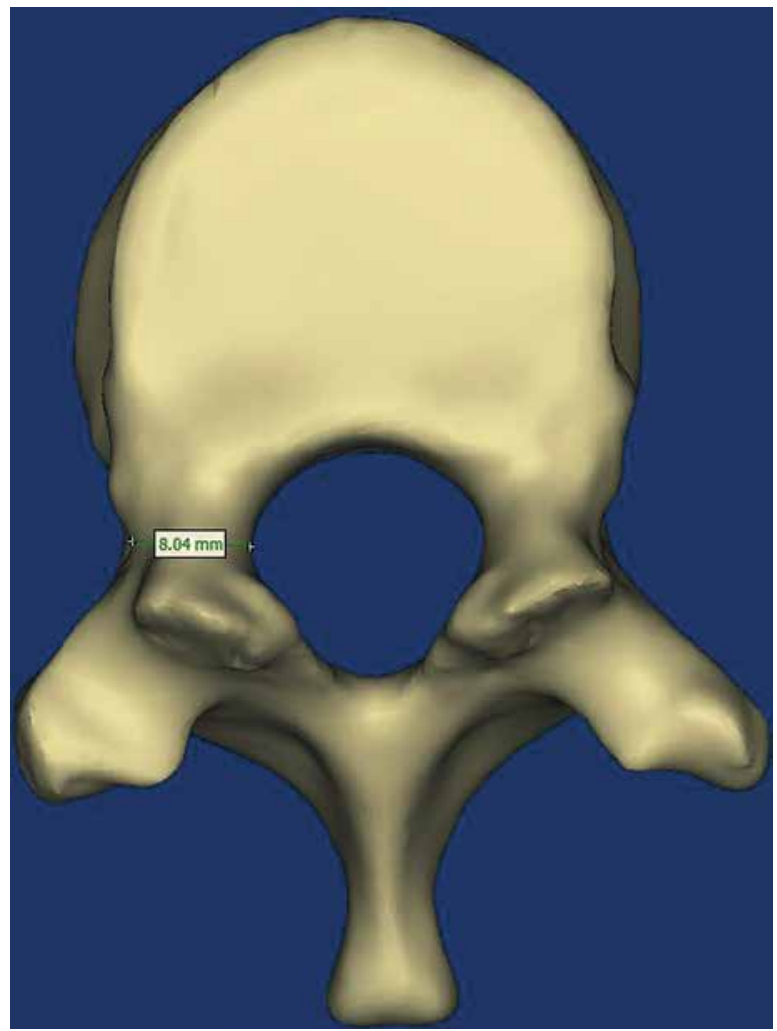

Photo 3. Pedicle width (PW) 


\section{Statistical analysis}

SPSS13.0 software was used for the statistical analysis; the data were expressed as $\bar{x} \pm s$; the left and right data of each parameter as well as the data between boys and girls were subjected to the $t$ test, and the paired-sample $t$ test was performed on the paired measurement data. Variance analysis was performed on the changes of the same measurement object among different vertebral sequences with the test standard $\alpha=0.05$ and $p<0.05$ considered as statistically significant.

\section{Results}

\section{Measurement results of ID and SD}

ID and SD showed no significant difference between the sexes or spinal sides, so they were combined for the statistics. In different age groups, ID showed a general increasing trend with the increasing vertebral sequence, namely $\mathrm{L} 5>\mathrm{L} 4>\mathrm{L} 3>\mathrm{L} 2>\mathrm{L} 1$, group $A$ : significant differences were seen between $\mathrm{L} 1$ and L2-5, L2 and L3-5, L3 and L4-5, and L4 and L5 ( $p<0.05$ ); group B: L1 and L2-5, L2 and L4-5, and L3-4 and L5 showed significant differences $(p<0.05)$, but the rest showed no significant difference $(p>0.05)$. ID showed an increasing trend with increasing age, namely, group $B>A$ group, and the intergroup data of L1-5 showed significant differences $(p<0.05)$; SD showed a general slow increasing trend in different age groups with the vertebral sequence increasing; in group $\mathrm{A}$, there were significant differences between L1 and L4-5, and L4 and L5 $(p<0.05)$; in group B, L1 and L3-5, L2 and L4-5,

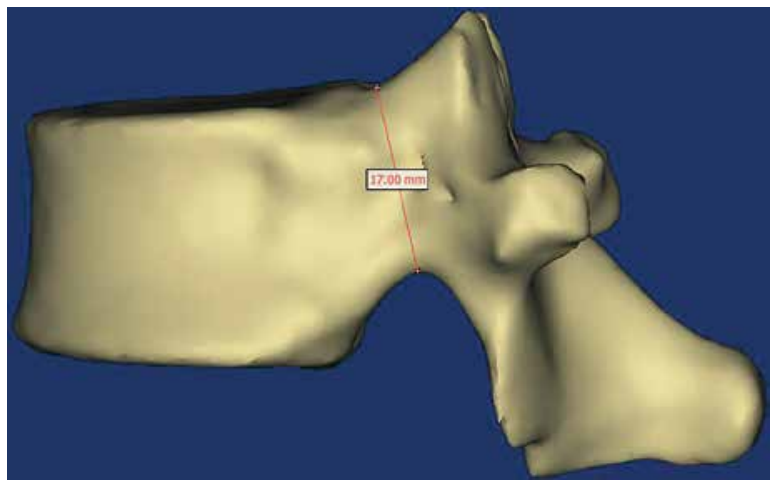

Photo 4. Pedicle height $(\mathrm{PH})$

and L3 and L4-5 showed significant differences $(p<0.05)$, and SD also increased with increasing age, and the intergroup data of L1-5 showed significant differences $(p<0.05)$ (Table II, Figure 1 ).

\section{Measurement results of $\mathrm{PW}$ and $\mathrm{PH}$}

PH and PW showed no significant difference between sexes or spinal sides, so they were combined for the statistics. PW showed increasing trends with increasing age and vertebral sequence; L1 and L45 , and L2-4 and L5 in group A, and L1-2 and L3-5, $\mathrm{L} 3$ and $\mathrm{L} 4-5$, and $\mathrm{L} 4$ and $\mathrm{L} 5$ in group $B$ showed significant differences $(p<0.05)$. As for different age groups, PW showed a significant intergroup difference between L1 and L3-5 $(p<0.05)$. PH showed a slow decreasing trend with the increasing vertebral sequence, in which group A showed no significant difference among different vertebra, but group $B$ showed a significant difference between $\mathrm{L} 3-5$ and L4-5 $(p<0.05)$, and the rest had no significant dif-

Table II. ID and SD measurements ( $\bar{x} \pm$ SD, min.- $\max .[\mathrm{mm}], n=60)$

\begin{tabular}{|lcccc|}
\hline Vertebral sequence & \multicolumn{2}{c|}{ Group A } & \multicolumn{2}{c|}{ Group B } \\
\cline { 2 - 5 } & ID & SD & ID \\
\hline L1 & $18.32 \pm 4.78^{*}$ & $13.01 \pm 3.38^{*}$ & $21.06 \pm 6.91$ & $17.97 \pm 5.74$ \\
& $(14.16-22.32)$ & $(9.57-16.01)$ & $(17.03-24.21)$ & $(13.08-21.37)$ \\
\hline L2 & $20.46 \pm 3.98^{* a}$ & $13.57 \pm 4.19^{*}$ & $23.18 \pm 6.29^{\mathrm{a}}$ & $19.18 \pm 4.38$ \\
& $(16.63-25.13)$ & $(10.22-16.27)$ & $(18.14-27.10)$ & $(14.12-23.39)$ \\
\hline L3 & $22.35 \pm 3.25^{* a b}$ & $13.93 \pm 5.16^{*}$ & $24.52 \pm 7.19^{\mathrm{a}}$ & $20.03 \pm 4.15^{\mathrm{a}}$ \\
& $(17.18-25.21)$ & $(10.83-16.24)$ & $(19.24-28.19)$ & $(17.03-25.21)$ \\
\hline L4 & $24.28 \pm 5.58^{* a b c}$ & $14.01 \pm 3.07^{* a}$ & $26.28 \pm 7.41^{\text {ab }}$ & $21.24 \pm 3.96^{\mathrm{abc}}$ \\
& $(19.28-28.41)$ & $(11.08-15.29)$ & $(23.03-30.08)$ & $(17.28-27.25)$ \\
\hline L5 & $26.01 \pm 5.55^{* a b c d}$ & $14.66 \pm 3.85^{* a b}$ & $28.95 \pm 6.99^{\mathrm{abcd}}$ & $21.85 \pm 5.01^{\text {abc }}$ \\
& $(20.18-31.23)$ & $(12.99-20.02)$ & $(25.59-35.51)$ & $(19.26-29.69)$ \\
\hline
\end{tabular}




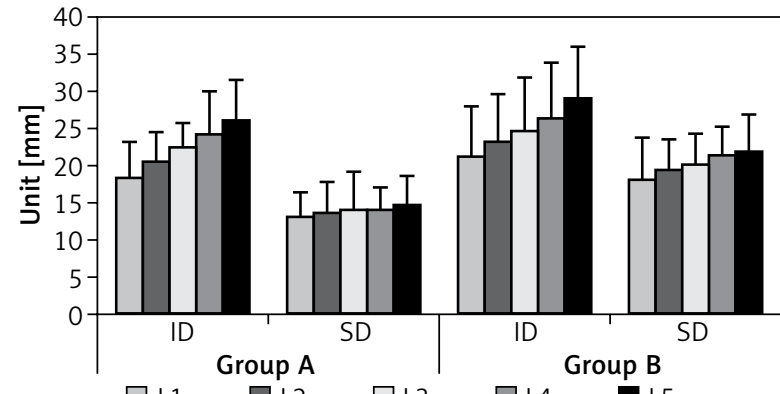

$\square \mathrm{L} 1$

$\square \mathrm{L} 2$

$\square \mathrm{L} 3$

$\square\llcorner 4$

L5

Figure 1. Cylindrical chart of pedia-lumbar ID and SD

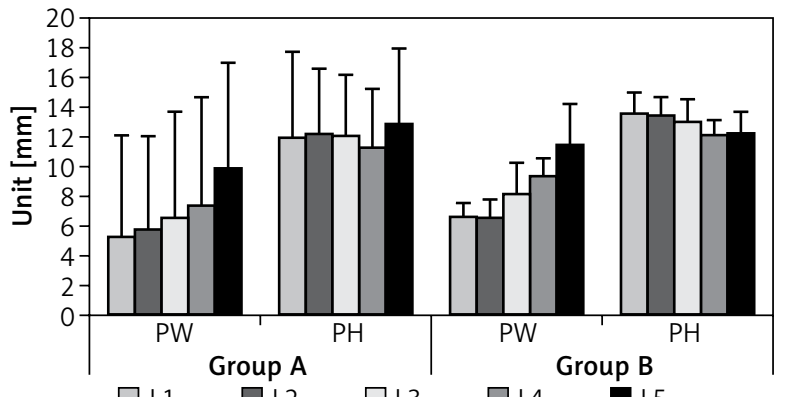

$\square \mathrm{L} 1$ $\square \mathrm{L} 3$

Figure 2. Cylindrical chart of pedia-lumbar PW and $\mathrm{PH}$

Table III. PW and PH measurements ( $\bar{x} \pm$ SD, min.-max. [mm], $n=120)$

\begin{tabular}{|lcccc|}
\hline Vertebral sequence & \multicolumn{2}{c}{ Group A } & \multicolumn{2}{c|}{ Group B } \\
\cline { 2 - 5 } & PW & PH & PH \\
\hline L1 & $5.23 \pm 6.90^{*}$ & $11.97 \pm 5.74^{*}$ & $6.62 \pm 0.91$ & $13.54 \pm 1.41$ \\
& $(3.03-6.21)$ & $(9.08-12.37)$ & $(6.03-7.21)$ & $(12.12-15.11)$ \\
\hline L2 & $5.75 \pm 6.29$ & $12.18 \pm 4.38^{*}$ & $6.49 \pm 1.26$ & $13.43 \pm 1.26$ \\
& $(4.14-6.10)$ & $(10.12-14.39)$ & $(6.10-7.26)$ & $(11.88-14.29)$ \\
\hline L3 & $6.52 \pm 7.19^{*}$ & $12.03 \pm 4.15^{*}$ & $8.15 \pm 2.17^{\mathrm{ab}}$ & $13.06 \pm 1.40$ \\
& $(4.24-7.19)$ & $(10.03-15.21)$ & $(7.00-9.01)$ & $(11.03-14.68)$ \\
\hline L4 & $7.28 \pm 7.41^{* a}$ & $11.24 \pm 3.96$ & $9.37 \pm 1.15^{\text {abc }}$ & $12.07 \pm 1.07^{\mathrm{abc}}$ \\
& $(5.03-8.08)$ & $(9.28-13.25)$ & $(7.88-10.22)$ & $(10.29-9.87)$ \\
\hline L5 & $9.95 \pm 6.99^{*}$ abcd & $12.85 \pm 5.01$ & $11.50 \pm 2.73^{\mathrm{abcd}}$ & $12.18 \pm 1.45^{\mathrm{abc}}$ \\
& $(7.59-11.51)$ & $(10.26-14.69)$ & $(9.12-12.21)$ & $(11.05-14.48)$ \\
\hline
\end{tabular}

Note: compared with group $B,{ }^{*} p<0.05$; compared with $L 1,{ }^{a} p<0.05$; compared with $L 2,{ }^{b} p<0.05$; compared with $L 3,{ }^{c} p<0.05$; compared with $L 4,{ }^{d} p<0.05$.

ference. Among the different age groups, $\mathrm{PH}$ showed a significant intergroup difference in L1-3 $(p<0.05)$, and generally, PH was bigger than PW $(p<0.05)$. PW and $\mathrm{PH}$ also increased with increasing age (Table III, Figure 2).

\section{Discussion}

\section{Feasibility analysis of lumbar diameter and spinal screw implantation}

The spinal canal is co-composed of the posterior lumbar edge, intervertebral disk, pedicle, vertebral lamina, and ligamentum flavum. The lumbar spinal canal connects upwards to the thoracic spinal canal and is bilaterally connected to the lumbar nerve root canal. Generally, the lumbar spinal canal is divided into the central canal, lateral crypts, and neural tube, and its contents include the spinal dura, arachnoid, cerebrospinal fluid, spinal conus, and coccygeal nerve. The space between the spinal dural and spinal canal wall is separated with loose con- nective tissue and fat, and the epidural space has epidural fat, arteries and veins, and nerve branches. The minimum gap between the spinal canal and contents allows the spinal contents to move freely without tension or stress. Therefore, the diameter of the spinal canal has important meaning for the clinical judgment of spinal development, spinal stenosis, or pedicle screw implantation. However, the current studies are more concentrated on adults [15], and a certain Chinese researcher [16], who observed 100 cases of adult lumbar spinal specimens, concluded that the spinal ID in both men and women showed an increasing trend with the increasing vertebral sequence. Some foreign scholars reported $[17,18]$ that the overall trends were similar, but different races had different spinal IDs, which might be related to ethnicities, geographies, or sexes. Based on the imaging and 3D reconstruction measurements towards the lumbar spines of 60 children aged 6-11 years, it was found that children exhibited a similar trend of spinal canal as that in adults, 
namely ID and SD gradually increased from $L 1$ to $L 5$, and this was associated with the gradually from-topto-bottom increasing lumbar loads, so it was consistent with its load-carrying functions. Its size was inversely proportional to the distance from the line of gravity, suggesting that clinical pedicle screw fixation in children can refer to the characteristics of that in adults as well as combining the development features and related parameters of the lumbar spine in children to achieve individualized screwing so as to reduce the incidence of surgical complications.

\section{Definition of child pedicle and feasibility analysis of pedicle screw implantation}

Spinal diseases in children are not the same as those in adults because children's spinal columns are at the peak of continuous development; meanwhile, children's thoracic and lumbar pedicles are smaller than those in adults, so the screwing operations have more technical difficulties and bigger risks; furthermore, lumbar screws specialized for children are rare. The impacts of the screws on the growth and development of the spine also need further studies. The pedicle is an important structure connecting the anterior and posterior spinal columns and is the strongest point connecting the posterior annexes and the vertebrae. Therefore, it is called the "force nuclear" by some scholars. The pedicle is the mechanical bridge connecting the vertebrae and the vertebral arch, and so it is the basis of posterior spinal anatomical fixation. Despite the LVP being thicker, the surrounding anatomical structures are not as complex as those around the cervicothoracic spine, and though pedicle screw implantation is relatively easy and safe, it is common to see clinical reports of such complications as improper positioning of pedicle screws causing nerve damage and pedicle screw loosening. The pedicles formed at different vertebral segments vary, and studies on the adjacent relationships of thoracolumbar structures and pedicles are also numerous. Based on the anatomical characteristics of the pedicle, the implanted screws must be only located on one correct channel in the 3D space. Zindrick et al. [19] pointed out that if the screw caused the rupture of the lateral pedicle cortex, it would reduce its stability, thus intero-inferiorly breaking out of the pedicle, entering the spinal canal easily, and causing serious consequences. Breaking out of the pedicle intero-inferiorly might irritate or directly damage the nerve roots distributed in the lateral crypts and neural holes, thus causing corresponding symptoms. If the depth and location of the implant is not accurate, the screw might break out from the front part, and then it might damage the anterior great vessels or abdominal organs. This study found that PH was bigger than PW, so in clinical pedicle screw implantation, the main bottleneck lies in PW. After implantation, the pedicle screws are mainly located at the pedicle cortical bone and the compressed cancellous bone. If the cortical bone does not rupture at this time, the screw stability would be the best. However, if the pedicle cortical bone is thinner and PW is smaller, fracture of the pedicle cortex might occur during screw implantation with the screw rotating forward causing extrusion [20].

The number of samples collected in the earlier period of this study was relatively small, so it can not really reflect the difference between genders. However, it is certain that the development is different between genders. As the sample size gradually increases, such a difference can be reflected. We keep on collecting and measuring the follow-up image data, which will be more persuasive as the sample size increases, thus guiding clinical applications.

\section{Conclusions}

Through this 3D reconstructional measurement of children's lumbar spines, as well as the trend that the transverse and sagittal diameters and the pedicle width of children's lumbar vertebral canal increase with age and increasing vertebral order, this study not only objectively reflects the development rules of children's spinal column but also provides a theoretical basis for pedicle screw fixation in children.

\section{Acknowledgments}

The National Natural Science Foundation of China $(81260269,81560348,81660358)$; the Inner Mongolia Autonomous Region University of Science and Technology research grant (NJZC16108); Inner Mongolia Medical University Science and Technology project (2015YKDKJBW03); Inner Mongolia Medical University million technology projects (YKD2017KJBW009).

\section{Conflict of interest}

The authors declare no conflict of interest. 


\section{References}

1. Menger RP, Storey CM, Nixon MK, et al. Implantation of C1 pedicle screws using minimal exposure: radiographic, clinical, and literature validation. Int J Spine Surg 2015; 9: 43.

2. Mendelsohn D, Dea N, Lee R, et al. Bilateral pedicle and crossed translaminar screws in C2. Asian Spine J 2015; 9: 783-8.

3. Zhang Z, Mu Z, Zheng W. Anterior pedicle screw and plate fixation for cervical facet dislocation: case series and technical note. Spine J 2016; 16: 123-9.

4. Stulik J, Vyskocil T, Sebesta P, et al. Atlantoaxial fixation using the polyaxial screw-rod system. Eur Spine J 2007; 16: 479-84.

5. Mueller TL, Miller NH, Baulesh DM, et al. The safety of spinal pedicle screws in children ages 1 to 12. Spine J 2013; 13: 894-901.

6. Ruf M, Harm J. Pedicle screws in 1-and 2-year-old children: technique, complication, and effect on further growth. Spine (Phila Pa 1976) 2002; 27: 460-6.

7. Fujimori T, Yaszay B, Bartley CE, et al. Safety of pedicle screws and spinal instrumentation for pediatric patients: comparative analysis between 0- and 5-year-old, 5- and 10-year-old, and 10- and 15-year-old patients. Spine 2014; 39: 541-9.

8. Chan CY, Kwan MK, Saw LB. Safety of thoracic pedicle screw application using the funnel technique in Asians: a cadaveric evaluation. Eur Spine J 2010; 19: 78-84.

9. Ruf M, Harms J. Posterior hemivertebra resection with transpedicular instrumentation: early correction in children aged I to 6 years. Spine (Phila Pa 1976) 2003; 28: 2132-8.

10. Platzer P, Jaindl $M$, Thalhammer $G$, et al. Cervical spine injuries in pediatric patients. J Trauma 2007; 62: 389-96.

11. Polly DW, Potter BK, Kuklo T, et al. Volumetric spinal canal intrusion: a comparison between thoracic pedicle screws and thoracic hooks. Spine 2004; 29: 59-63.

12. Wang S, Qiu Y, Liu W, et al. The potential risk of spinal cord injury from pedicle screw at the apex of adolescent idiopathic thoracic scoliosis: magnetic resonance imaging evaluation. BMC Musculoskelet Disord 2015; 16: 310.

13. Sarwahi V, Sugarman EP, Wollowick AL, et al. Prevalence, distribution, and surgical relevance of abnormal pedicles in spines with adolescent idiopathic scoliosis vs no deformity: a CT-based study. J Bone Joint Surg Am 2014; 96: e92.

14. Hedequist DJ, Hall JE, Emans JB. The safety and efficacy of spinal instrumentation in children with congenital spine deformities. Spine (Phila Pa 1976) 2004; 29: 2081-6.

15. Ferree BA. Morphometric characteristics of the immature spine. Spine (Phila Pa 1976) 1992; 17: 887-91.

16. Li XH, Cai YQ, Wang X, et al. Anatomical characteristics of infra-lamina ridge in Chinese population. Int J Morphol 2014; 32: 432-7.

17. Tacar O, Demirant A, Nas K, et al. Morphology of the lumbar spinal canal in normal adult turks. Yonsei Med J 2003; 44: 679-85.

18. Santiago FR, Milena GL, Herrera RO, et al. Morphometry of the lower lumbar vertebrae in patients with and without low back pain. Eur Spine J 2001; 10: 228-33.

19. Zindrick MR, Wiltse LL, Doornlk A, et al. Analysis of the morphometric characteristics of the thoracic and lumbar pedicles. Spine (Phila Pa 1976) 1987; 12: 160-6.

20. Chen KR, Liu SS. Anatomical investigation on the internal structure of lumbar pedicle. Sichuan J Anatomy 2007; 5: 27-8.

Received: 9.05.2018, accepted: 3.06.2018. 